\title{
The Material Reasoning of Folding Paper
}

Michael Friedman, Colin Rittberg ${ }^{1}$

This is a pre-print. Paper to appear in Synthese.

This paper inquires the ways in which paper folding constitutes a mathematical practice and may prompt a mathematical culture. To do this, we first present and investigate the common mathematical activities shared by this culture, i.e. we present mathematical paper folding as a material reasoning practice. We show that the patterns of mathematical activity observed in mathematical paper folding are, at least since the end of the $19^{\text {th }}$ century, sufficiently stable to be considered as a practice. Moreover, we will argue that this practice is material. The permitted inferential actions when reasoning by folding are controlled by the physical realities of paperlike material, whilst claims to generality of some reasoning operations are supported by arguments from other mathematical idioms. The controlling structure provided by this material side of the practice is tight enough to allow for non-textual shared standards of argument and wide enough to provide sufficiently many problems for a practice to form. The upshot is that mathematical paper folding is a non-propositional and non-diagrammatic reasoning practice that adds to our understanding of the multi-faceted nature of the epistemic force of mathematical proof. We then draw on what we have learned from our contemplations about paper folding to highlight some lessons about what a study of mathematical cultures entails.

\section{Introduction}

Many arguments found in mathematical proofs are wholly propositional. Others are partly diagrammatic. Recently, the philosophy of mathematical practice has studied diagrammatic reasoning in depth, ${ }^{2}$ showing that text and diagrams must be thought together to understand diagrammatic reasoning, without reducing one to the other. In this article we present and investigate a third type of mathematical reasoning: material reasoning. We present paper folding as an exemplar of this reasoning. Our aim is to inquire what makes paper folding a mathematical practice, how it is structured, how reasoning in this practice is carried out, and eventually how this activity enables a mathematical culture.

The practice of mathematical paper folding we focus on in this paper is the fold in which paper is folded flat onto itself and then opened up again. This creates a straight crease in the paper. Folding is thus an operation, which constructs a straight line on what we call the creasediagram: the unfolded piece of paper on which the network of straight line creases lies. One can also construct the perpendicular to a line (i.e. to a crease) by folding the paper such that the crease is folded onto itself. We can also fold two given intersecting lines, one on top of the other, constructing the angle-bisector (to the angle between these lines) as the crease. In this manner we construct lines in a controlled fashion and this allows for doing geometry with it. ${ }^{3}$

\footnotetext{
${ }^{1}$ Both authors have contributed equally to this work.

2 Examples include (Mancosu 2008); (Manders 2008); (Avigad et al. 2009); (Leitgeb 2009); (Larvor 2012); (De Toffoli and Giardino 2014); (Larvor 2016).

3 The use of a compass is prohibited in folding constructions and hence circles cannot be drawn. 
It turns out that geometry by folding constructions is surprisingly strong. By folding we can trisect an angle and double the cube, two problems famously unsolvable via straightedge and compass constructions alone. The reasoning involved in doubling the cube by paper folding will be presented in more detail later in this article. Folding points on lines or lines on lines are examples of basic folding operations. What should be considered as basic folding operation is, however, not obvious, and mathematicians have explored this in detail and (eventually) proved the generality of these folds (sections 4 and 5). This stands in contrast to the situation with axiomatic reasoning practices, where the permissible inferential actions are fixed at the outset - e.g. in Euclidean practice by the first three postulates. ${ }^{4}$

There are other types of paper folding, which might point to other styles of reasoning. Origami comes to mind, in which three-dimensional objects are created; another type of paper folding is the folding in which cuts are made to create two-dimensional objects. ${ }^{5}$ Furthermore, one can generalize mathematical paper folding by allowing more than one fold to be made simultaneously. This defies the physical limitations of paper; it is impossible to fold two creases that meet on a sheet of paper simultaneously; see (Alperin and Lang 2009). In this article, we do not investigate the reasoning involved in the mathematics in these other types of paper folding. Our focus is exclusively on the reasoning of mathematical paper folding which folds and then opens the paper up again in the way described above and we will refer to it from now on as folding.

One reason to investigate reasoning by folding is that it appears as a practice that is essentially different from diagrammatic reasoning. We aim to highlight some of these differences, thereby expanding our descriptive grip on mathematical reasoning practices. Once we have understood folding as a mathematical reasoning practice we can inquire if folding paper enables a mathematical culture, how it could do so and what this may mean for our understanding of the term "mathematical culture".

Mathematical reasoning practices have received some philosophical attention in recent years. One focus has been the role of 2-dimensional, drawn diagrams and sketches. Kenneth Manders' seminal paper “The Euclidean Diagram", written in 1995, begins as follows:

"In Euclidean geometry, a diagram has standing to license inference, just as do relationships recognized in the text. It is now commonly held that this is a defect of rigor." (Manders 2008, p. 80)

In his article, Manders argues that the rigor of Euclidean diagrammatic reasoning is, in fact, not defect. Other publications have appeared that aim to do for other diagrammatic reasoning practices in mathematics what Manders has done for the Euclidean diagram; (Carter 2010), (De Toffoli and Giardino 2014, 2016). This has bearing on the debate about validity and

\footnotetext{
${ }^{4}$ See (Larvor 2017). Explicitly, by the first three postulates we mean the following: 1 . A straight-line segment can be drawn joining any two points. 2. Any straight-line segment can be extended indefinitely in a straight line. 3. Given any straight-line segment, a circle can be drawn having the segment as radius and one endpoint as center.

${ }^{5}$ See for example: (Demaine et al 1999).
} 
rigor in mathematical proofs. Manders' account and the subsequent works in history and philosophy of mathematical practices have challenged the view that diagrammatic reasoning can be translated and reduced completely into propositional, formalistic reasoning. These works challenge $20^{\text {th }}$ century modernism "with its preference for general theory, symbolic formalism, and the treatment of mathematical theories as worlds of their own without any immediate relation to the physical world around us", being closely related to the "the neglect of 3-D models (and 2-D diagrams) during most of the twentieth century." (Mehrtens 2004, p. 278)

According to De Toffoli and Giardino, diagrammatic reasoning is to be viewed as essentially informal in the sense that its 'translation' to syntactical propositions distorts the knowledge transmitted through it; formalizing diagrammatic reasoning not only alters the arguments made via the diagrams but also changes what the reasoning acts on. In contrast to the diagrammatic reasoning, reasoning by folding, especially from the end of the $19^{\text {th }}$ century onwards, has developed alongside the formalization of mathematical reasoning. We will highlight the interplay between formal and informal reasoning idioms in the folding practice. Folding is not essentially informal in the sense which the above-mentioned scholars attribute to diagrammatic reasoning, in part because, as we will show, the practitioners of the folding practice sometimes reason in a fully propositional-syntactic reasoning idiom.

The differences between diagrammatic reasoning and folding based reasoning cannot be reduced to the different approaches concerning formalization. To emphasize, the creasediagram is only an end-product of an inferential process when reasoning by folding. The haptic dimension of moving the two-dimensional plane depicted in the crease-diagram through threedimensional space to align points and lines plays a fundamental role in the epistemic force of proofs by folding. Folding, we will argue, is not a diagrammatic reasoning practice, since the rationality of a folding proof is carried, in part, by the process of folding the crease-diagram. It is also not a purely propositional reasoning practice. We suggest naming it by the source of its epistemic force: a material reasoning practice. This practice is what stands, as we aim to show, at the basis of a mathematical culture of folding.

Let us briefly review the structure of our paper. Section 2 gives some preliminary thoughts on practices, which will guide the discussion and suggest why folding is a mathematical reasoning practice. We present a brief survey of the history of paper folding, concentrating mainly (but not solely) on the first third of the $20^{\text {th }}$ century, starting from Tandalam Sundara Row's 1893 book Geometrical exercises in paper folding.

In section 3 we discuss how mathematicians practise folding. We discuss three examples to make visible how the haptic operations of folding and symbolic-propositional idioms interact when reasoning via paper folding.

We engage in section 4 in more detail with the basic actions of reasoning by paper folding. We discuss the basic folding operations and draw attention to how the mathematicians came to know them, showing that the permissible inference steps of reasoning by paper folding are not given to us propositionally at the outset and that inquiring what these permissible inferential steps are is part of the practice.

In section 5 we move to a discussion of the epistemic force of proofs by folding. We present Giaquinto's ahistorical account of this epistemic force as a means to access the interplay of reasoning idioms one finds in the folding practice. 
Bringing together the insights of the earlier sections, section 6 argues that folding is a material reasoning practice. We highlight some differences with other non-propositional reasoning practices that have been discussed in the literature. We argue that, unlike in the cases of diagrammatic reasoning, in the case of paper folding the physical realities of the material reasoned on ensure the epistemic force of arguments. ${ }^{6}$

Having clarified the questions concerning practices, we turn to the question of mathematical cultures and folding in section 7 . We deal directly with the culture terminology and draw attention to discontinuities of folding as a culture with some classical understandings of the term. Rather than arguing that folding based geometry is or is not a mathematical culture, we propose to learn from our contemplations that mathematical cultures need not be based on shared beliefs but rather on shared practices.

\section{Paper folding as a mathematical practice: short historical review}

Part of our aim is to inquire what structures folding as a reasoning practice and how reasoning in this practice is carried out. But before doing that, we need to tackle the question whether one can meaningfully speak about a practice of paper folding.

De Toffoli and Giardino (2016, p. 28) give us a three-item list of what a philosophy of mathematical practice should investigate. From this we take that a mathematical practice is a human activity, which contains a collective dimension in the sense that a mathematical community exists, certain mathematical capacities are enhanced via this activity and material representations are being used. While the last aspect is obvious with respect to paper folding, the first and the second require elaboration. Hence, we will give in this section a brief historical survey of folding to argue that folding is a mathematical practice. A full blown historical analysis of the development of mathematical paper folding, starting from the $16^{\text {th }}$ century till the end of the $20^{\text {th }}$ century, can be found in the first author's upcoming book A History of Folding in Mathematics: Mathematizing the Margins; (Friedman 2018). Here we will review only the main mathematicians working in the late $19^{\text {th }}$ century and the $20^{\text {th }}$ century.

Joseph Rouse notes that there should be sufficiently many practitioners for folding to be considered as a practice:

Practices are patterns of activity in response to a situation. Practices are dynamic, because these patterns exist only through being continually reproduced. Their coherence and continuity thus depend both on coordination among multiple participants and things and on the maintenance of that coordination over time. (Rouse 1996, p. 26, emphasis added),

To show the existence of the "multiple participants", i.e. of a community of mathematicians who practised folding in their work, we begin our discussion in 1893. In this year, the Indian mathematician Tandalam Sundara Row published his book Geometrical Exercises in Paper Folding (Row 1893), presenting many exercises and theorems that can be solved and proved

\footnotetext{
${ }^{6}$ Having motivated our philosophical interest in folding, allow us now to point out the joy that comes with paper folding. We invite our readers to read this paper with a sheet of semi- transparent paper (baking paper works nicely) and a pencil to hand to perform some of the folding we will discuss in later sections.
} 
using solely the means of paper folding. In fact, Row attempted to base all of plane geometry on operations stemming from folding. ${ }^{7}$ Row wrote:

The teaching of Euclid in schools can be made very interesting by the free use of the kindergarten gifts. It would be perfectly legitimate to require pupils to fold the diagrams on paper. This would give [the children] neat and accurate figures, and impress the truth of the propositions forcibly on their minds. It would not be necessary to take any statement on trust. (Row 1893, p. ii)

Row suggests that there would not be any need to consider axioms as an unprovable basis without justification (the "statement on trust"). Row also indicates his sources of influence: he starts the introduction of his book by referring to kindergarten gifts. This indicates that Row was inspired by the exercises and playful objects given to children at the kindergartens of Friedrich Fröbel (called "gifts and occupations"); indeed, Row indicates that "[t]he idea of this book was suggested to me by Kindergarten Gift No. 8. -Paper-folding. The gift consists of 200 variously coloured squares of paper [...] and diagrams and instructions for folding." 8 But while the Fröbelian exercises were meant for young children, Row took Fröbel's ideas one step further, ${ }^{9}$ as we will see in more detail in section 4.1 . Indeed, in his book, Row did not only construct via folding several regular polygons, but also dealt with arithmetic, geometric, and harmonic series, properties related to the concurrence of lines and more importantly - the construction of conic sections and other curves of higher degree. ${ }^{10}$

Row's book became influential. Two years after the publication of the book, Felix Klein mentions it in his 1895 book Vorträge über ausgewählte Fragen der Elementargeometrie: ${ }^{11}$

"[...] a Hindu mathematician, Sundara Row, of Madras, published a little book Geometrical Exercises in Paper Folding, in which [this] idea is considerably developed. The author shows how by paper folding we may construct by points such curves as the ellipse, etc." 12

Klein's book was translated in 1897 to English and his remark has sparked an interest, causing a republication of Row's book in the USA by Wooster Woodruff Beman and David Eugene Smith. In 1901 a new edition of Row's book was published in Chicago as (Row 1901), where the editors remark that they were enthusiastic regarding the novel methods presented there.

Row's book and its re-publication have caused a popularization of the mathematization of paper folding. Several mathematicians were influenced by his work and tried to advance it

\footnotetext{
7 For a survey on Row's work and on Hermann Wiener's work, another mathematician who worked with folded models in 1893, see: (Friedman 2016).

8 (Row 1893, p. i). Note that Eleonore Heerwart, an influential educator who helped spreading the Fröbelian ideas in Great Britain in the second half of the $19^{\text {th }}$ century, numbered paper folding as gift num. 8 (Friedman 2018, section 4.2.1.3). The Fröbelian ideas came to India in the wake of Great Britain's colonization activities, which indicates how Row may have encountered them. See: (Friedman 2018, section 4.2.2).

9 See also: (Friedman 2018, section 4.2.1.2).

10 By this we mean that by using folding techniques Row was able to construct an infinite number of points that lie on the given curve, hence constructing the curve as the geometrical place of these points. Obviously, the only curve that can be constructed without gaps with paper folding is a line.

11 It is still not clear how Klein obtained Row's book.

12 (Klein 1895, p. 33). The translation is taken from (Klein 1897, p. 42). 
further. For example, Wilhelm Ahrens (1872-1927), Adolf Hurwitz (1859-1919), and C. A. Rupp were all influenced by Row's work. Each placed folding in a different mathematical culture and domain: Ahrens in recreational mathematics, in his 1901 book Mathematische Unterhaltungen und Spiele; (Ahrens 1901); Hurwitz emphasizing in his private diaries the constructions which can be made with folding; ${ }^{13}$ and Rupp investigating envelopes of curves in his 1924 paper "On a Transformation by Paper Folding." (Rupp 1924). Another work - the 1905 book A First Book of Geometry of Grace Chisholm Young and William Young - took a less favorable approach to Row's work (Young and Young 1905). This book clarifies, in contrast to Row, the distinction between the fold as operation and the (Euclidean) geometrical axioms. The fact that the book was quite popular in Europe also helped spreading folding as mathematical practice.

It was however with the work of the Italian mathematician Margherita Piazzolla Beloch (1872-1976), who was familiar with Row's work as well as with Rupp's paper, that a breakthrough occurred. Her work showed that certain constructions can be obtained via foldingbased geometry, while the same constructions are not obtainable by straightedge and compass constructions alone. ${ }^{14}$ Beloch showed that one of the Delian problems - doubling the cube, which boils down to constructing a segment whose length is the third root of $2-{ }^{15}$ can be solved by paper folding. Beloch's fundamental insight in her (1934) was that the following operation can always be performed, ${ }^{16}$ for any two points $\mathrm{P}_{1}$ and $\mathrm{P}_{2}$ and two lines $\mathrm{l}_{1}$ and $\mathrm{l}_{2}$ :

Beloch fold: Given two points $\mathrm{P}_{1}$ and $\mathrm{P}_{2}$ and two lines $\mathrm{l}_{1}$ and $\mathrm{l}_{2}$ one can make a single fold that places $\mathrm{P}_{1}$ onto $\mathrm{l}_{1}$ and $\mathrm{P}_{2}$ onto $\mathrm{l}_{2}$ simultaneously.

We will return to Beloch's folding below, but it will already be helpful here to examine how she constructed a segment whose length is the $\sqrt[3]{ } 2$. Beloch took the points $A=(-1,0)$ and $B=$ $(0,-2)$ and folded them onto the lines $x=1$ and $y=2$ respectively. She then proved that the intersection of the obtained crease (the dotted line in figure 1) with the $\mathrm{y}$-axis is the point $\mathrm{C}=$ $\left(0,{ }^{3} \sqrt{2}\right) \cdot{ }^{17}$

\footnotetext{
${ }^{13}$ On Hurwitz work on folding, see: (Oswald 2015). On the connection between Ahrens and Hurwitz conceptions of folding, see also (Friedman 2018, section 5.1.1).

14 See (Hull 2011). See also (Friedman 2018, section 5.2.2).

${ }^{15}$ To double the volume of a cube of side-length 1 one needs to construct a cube of side-length third root of 2 .

${ }^{16}$ Note that Beloch's claim, that the operation can always be performed, requires the piece of paper to be indefinitely large. We discuss this requirement in Section 4.1

${ }^{17}$ To see this, we follow the argument in (Hull 2011, pp. 310-311): Denote by M the intersection of the fold with the $\mathrm{x}$-axis. Using properties of similar triangles, we obtain:
}

$$
|\mathrm{OC}| /|\mathrm{OA}|=|\mathrm{OM}| /|\mathrm{OC}|=|\mathrm{OB}| /|\mathrm{OM}|
$$

Substituting $|\mathrm{OA}|=1$ and $|\mathrm{OB}|=2$ we obtain:

$$
|\mathrm{OC}|=|\mathrm{OM}| /|\mathrm{OC}|=2 /|\mathrm{OM}|
$$

Hence

$$
|\mathrm{OC}|^{3}=|\mathrm{OC}| \cdot|\mathrm{OM}| /|\mathrm{OC}| \cdot 2 /|\mathrm{OM}|=2
$$




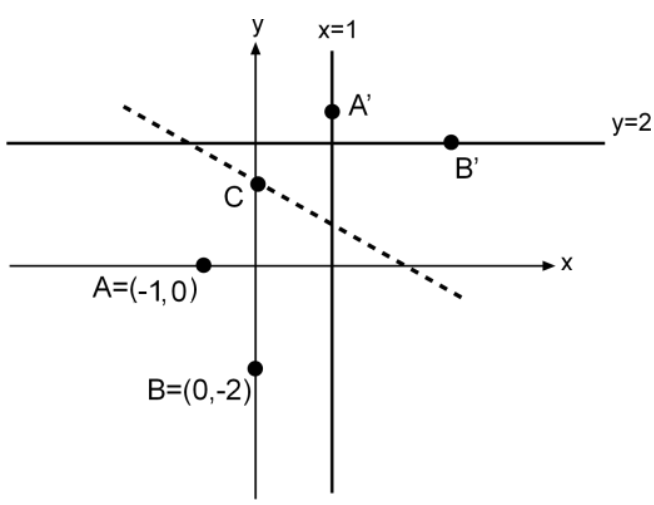

Figure 1: Beloch's construction on the third root of 2. (figure drawn by M.F.)

Today, Beloch's 1934 constructions are seen as a mathematical breakthrough. However, at the time they were ignored and forgotten ${ }^{18}$ and were only rediscovered by Humiaki Huzita (19242005 ) in the middle of the 80 s of the $20^{\text {th }}$ century. They were republished in the 1989 conference proceedings "The First International Meeting of Origami Science and Technology". In these proceedings, Huzita and Jacques Justin published their own work on fundamental operations of folding based geometry; (Justin 1989 [1986]), (Huzita 1989). Other mathematicians, such as Emma Frigerio and Benedetto Scimemi also published in these proceedings important papers on this subject and on Beloch's work; (Friegerio and Huzita 1989), (Huzita and Scimeni 1989).

Our historical account reveals that folding has, after Row's seminal book, attracted sufficient attention for a community to form. The interests of the actors that led to this community forming are diverse. Row was interested in school teaching; Beloch was interested in the force of the mathematical constructions possible with folding (doubling the cube); Justin and Huzita were interested in many kinds of paper-folding (such as origami) and mathematical paper folding in the sense of this article was just part of this.

Rouse stressed "Practices are patterns of activity in response to a situation" (see citation above). The situation brought about by paper and the method of line and point constructions via folding is still with us. Some research mathematicians investigate the kind of contemporary questions that arise from the folding situation. ${ }^{19}$ Paper is also still folded as part of recreational and educational mathematics. ${ }^{20}$ Folding lives on as a mathematical practice because there are patterns of activity in response to the situation encountered in paper folding.

The above non-exhaustive survey, concentrating mainly on the first decades of the $20^{\text {th }}$ century, shows the existence of a mathematical community dealing mathematically with paper folding, starting (at least) from the $19^{\text {th }}$ century. These mathematicians were taking part in a cooperative effort; indeed, for Manders, "at its most basic, a mathematical practice is a structure for cooperative effort in control” of communal mathematical activities; (Manders 2008, p. 82). ${ }^{21}$

\footnotetext{
${ }^{18}$ The reasons for this ignorance are beyond the scope of this article. For a detailed analysis of these reasons see: (Friedman 2018, section 5.2.3).

${ }^{19}$ Examples include (Bern and Hayes 1996), (Bern et al. 2003), (Demaine and O'Rourke 2007) and (Alperin and Lang 2009).

${ }^{20}$ See for example Vi Hart's famous video on YouTube from 2011 on the proof of the Pythagorean theorem via folding (https://www.youtube.com/watch?v=z61L83wl31E) and Matthew Stone and Una Stojnic analysis of it (Stone and Stojnic 2015).

21 For the diagrammatic reasoning, an example of this control is, according the Manders, that "practice has to control the production of diagrams and provide for resolution of disagreement among judgments based on their appearances" (ibid., p. 91). 
This resonates with Rouse's statement, that: "what scientists share is not a background of beliefs but a situation that they may belong to and understand in partially different ways" (Rouse 1996, p. 27). ${ }^{22} \mathrm{We}$ will argue that for folding this controlling structure (or situation) is given largely by the physical realities of paper. We say 'largely' here because, as we will see, the arguments for generality of some folds and folding operations given by the mathematicians rely on other mathematical idioms, practices and traditions. These matters will be discussed in more detail in the following sections. An investigation how this structure controls and regulates the activities of the mathematicians extends over sections 4 and 5. Viewed in this light, our argument that folding is a practice only ends with the end of this article.

Before turning to a more philosophical analysis, we need to look more closely at a few case studies.

\section{Three case studies}

In this section we provide three short case studies on how reasoning by folding was done. Two are taken from Row, one is due to Beloch. Our aim is twofold. First, this section will provide the reader with insights on how folding was practised. As we will see, Row and Beloch practised folding in very different ways. Second, we will use the discussion of our case studies to draw out two features of reasoning via paper folding - idealization and generalization - which will be philosophically analyzed in the next sections.

\subsection{Row's geometric series}

Row starts his book with the folding of a square. To obtain such a square Row takes any piece of paper, folds it upon itself, thus obtaining a crease. Folding the crease onto itself, he obtains a second crease, which is perpendicular to the first one. Continuing in that way to form more perpendicular creases, he obtains a rectangle; indeed, for Row, "by superposition, it is proved that $[\ldots]$ the two long sides [of the rectangle] are equal, and so also are the two short sides." (Row 1893, pp 1-2) We consider what can be either proved or read off from a folding diagram in section 5.1.

Row now performs the decisive step: "Now take this rectangular piece of paper, and fold it obliquely so that one of the short sides falls upon one of the longer sides. Then fold and remove the portion which overlaps. Unfolding the sheet, we find that it is now square, i.e., its four angles are right angles, and all its sides are equal." (p. 2) For what follows, the length of these sides is considered to be 1 . Row then folds along the second diagonal of the square, noting that the point of intersection of the two diagonals is the mid-point of the square (p. 5) By folding each pair of parallel edges on its opposite side, Row obtains four smaller squares. He then folds "through the corners of the smaller squares, [...] [to] obtain a square [which] is half the larger square, and has the same center" (p. 4). ${ }^{23}$ With "the corners of the smaller squares" Row means the corners lying on the middle of the edges of the large square. With this smaller square Row

\footnotetext{
${ }^{22}$ See also (Netz 2017) on this theme. See (Netz 1999) for a full account.

${ }^{23}$ Note that this step this is similar to what Giaquinto considers while discussing folding as proof practice; see: (Giaquinto 2007, p. 51), though he does not mention Row. We will return to the differences between Row's and Giaquinto's approach later (see section 5). Notice here that whilst Giaquinto also folds through mid-points, Row's prior folding operations have constructed these mid-points.
} 
performs the same procedure of folding the corners onto the center of it, obtaining now a square whose area is $1 / 4$. Performing this procedure again and again, he concludes:

"Each square leaves $1 / 2$ of the next larger square, i.e., the four triangles left from each square are together equal to half of it. The sums of all these triangles increased to any number cannot exceed the original square, and they must eventually absorb the whole of it." (Row 1893, pp. 4-5)

Since the sum of the areas of the folded triangles is eventually equal to the area of the whole original square, one obtains the following formula: $0.5+0.25+0.125+\ldots=1$. Row considers this to be a proof by folding of an infinite sum; see figure 2 for Row's crease-diagram after the third stage.

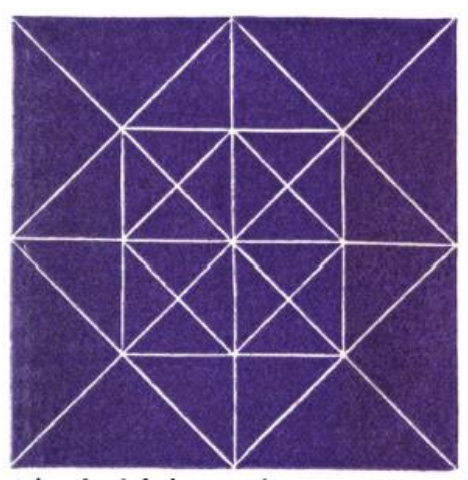

Figure 2: Row’s square paper after three consecutive folds; (Row 1893, p. 4)

Notice that for Row there is no need to justify his formula via an algebraic-symbolic reasoning. The evidence of the convergence of Row's geometric series is provided via folding, and it does not rely on other mathematical practices or traditions (such as algebra); (Row 1893, pp. 4-5). As we will see below, not all mathematicians who reason by paper folding follow Row in this. That Row sees no need to further justify the convergence of the series indicates that he regards folding not just a means of acquiring a belief ${ }^{24}$ but as a valid form of reasoning. That reasoning by folding can be more than a mere means to acquire beliefs has been challenged by Giaquinto who, roughly, argues that folding is not exact enough to carry validity. We will engage with this issue in section 5 .

\subsection{Row's construction of the parabola}

To further understand the reasoning underlying folding, we now turn to Row's connection between folding and outlining a parabola. Explicitly, given a line $l$ and a point $S$ not on $l$ (both given on a piece of paper), the various foldings of the line $l$ onto $S$ create creases which are tangent to the same parabola (see figure 3 ; the line $l$ is in figure 3 the bottom edge of the square); the obtained parabola has as a directrix the line $l$ and as a focus the point $S$, or, formulated

\footnotetext{
${ }^{24}$ We refer here to Giaquinto's terms "evidence-providing" and "non-evidential" (Giaquinto 2007, p. 67). We come back to Giaquinto in the next sections. 
otherwise, this parabola is the envelope $e^{25}$ of these creases; Row does not use this terminology, although it was well known during his time.

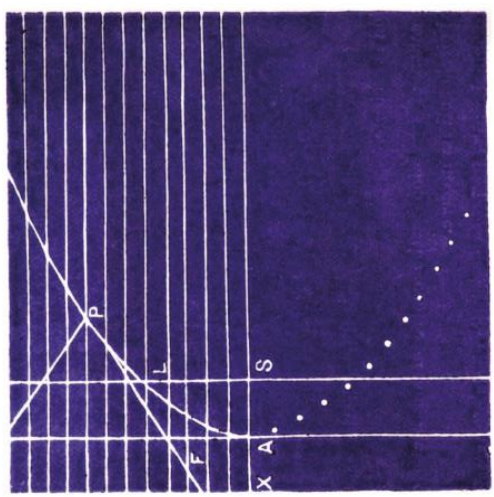

Figure 3: Row's drawing of a folding tangent to a parabola. (Row 1893, p. 88)

Row explains this connection as follows:

\begin{abstract}
"The above figure shows how a parabola can be marked on paper. The edge of the square $\mathrm{XF}$ is the directrix, A the vertex, and S the focus. Fold through XAS and obtain the axis. Divide the upper half of the square into a number of sections by lines parallel to the axis. These lines meet the directrix in a number of points. Fold by laying each of these points on the focus and mark the point where the corresponding horizontal line is cut. The points thus obtained lie on a parabola. The folding gives also the tangent to the curve at the point, e.g., PF." (Row 1893, p. 88)
\end{abstract}

In modern notation, if the point $\mathrm{X}$ (in figure 3 ) is $(0,0)$ and the point $\mathrm{S}$ is $(0, a)$ then the equation of the parabola is $y=x^{2}+a / 2$. Once again, the reasoning here is material: Row notes that the "parabola can be marked on paper". He uses the algebraic definition of the parabola as the set of all points, such that for any point $\mathrm{P}$ the distance $|\mathrm{PF}|$ to a fixed point $\mathrm{F}$, called the focus, is equal to the distance $|\mathrm{P} l|$ to a fixed line $l$, called the directrix. This definition enables Row to construct a set of points lying on the parabola materially via folding.

\title{
3.3 Beloch's fold
}

Having seen in the former subsection that folding a point on a line is equivalent to finding a tangent to a parabola, we will now show how Beloch used a multiplicity of practices and types of reasoning to justify the general existence of her fold (cf. section 1). These practices, we claim, were interwoven for Beloch. Using what we learned from Row in the last section and switching freely between reasoning idioms, we can see that proving that folding two different points on two different lines is always possible is equivalent to proving that a common tangent to two parabolas always exists. As Beloch mentioned in her 1934 article, given two points and two lines "it is enough [...] to fold the paper" in such and such way to find the desired (Beloch) fold, or that alternatively the "explained operation may be done with the same ease and accuracy with an ordinary drawing"; (Beloch 1934, p. 187). That is, for private cases, one may manipulate paper to see that the fold can indeed be performed. However, to justify the general

\footnotetext{
25 An envelope of a family of curves in the plane is a curve that is tangent to each member of the family at some point.
} 
case, i.e. that folding two different points on two different lines is always possible - Beloch turns towards another reasoning practice, based on computations from analytic geometry. ${ }^{26}$

As we have seen above, one can construct a parabola from a given line and a point not lying on the line. Row had already connected this insight to folding, as we saw in the last subsection. Beloch realized that she could use this connection to folding together with a theorem from analytic geometry to argue that what we now call the Beloch fold can always be performed. The theorem is that any two parabolas have at least one common tangent. Beloch's ingenious insight was that this common tangent is precisely the fold, which folds the two points onto the two lines as described in the Beloch fold. That this insight holds true requires proving, which Beloch did in the reasoning idioms of analytic geometry that were standard in her time.

This episode shows the ease with which Beloch moves between manual operations and symbolic idioms. This shows us something important about folding as a reasoning practice: reasoning by paper folding is multi-faceted and the idioms used intertwine in practice. When we claim that folding is a material reasoning practice we thus do not claim that all epistemic force of a proof by paper folding stems solely form the folds that can possibly be carried out on the material. Rather, what can possibly be folded using the material plays an important, but not the only, role in carrying epistemic force by, as we will argue, constraining allowed reasoning steps.

This episode also shows that mathematicians were interested whether certain folding actions can always be carried out. Indeed, Beloch's focus in her papers is on justifying why a certain fold - the Beloch fold - is an action, which can be carried out in general in the folding idiom. That this fold doubles the cube is presented as a side-product. Compare this to the simpler operation of folding any given generic point on any given generic line. Its general execution is evident without the need to justify it via a turn to another type of reasoning. That is, it is evident that this operation can always be performed and need not be proved via other means. And while for the Beloch fold - folding any two given generic points on any two given generic lines - the reasoning behind this execution is based on an interplay of material and propositional idioms stemming from analytic geometry, this might not be the case for more complicated versions of this operation. Indeed, the operation of folding three different points on three different lines is equivalent to finding a common tangent to three parabolas. But this tangent exists only in special cases. Justifying this general non-existence can be done only via reasoning stemming from analytic geometry, for example, but not from folding paper alone.

\section{Generic folding actions}

In the last section we drew attention to some features of reasoning by folding as it occurs in practice. We saw how Row's proof of an infinite sum asked us to ignore physical limitations such as how often a piece of paper can be folded. These are matters of idealization and we deal with them in the next subsection. We also met Beloch who took seriously the question whether certain folds can always be carried out. Such questions of generality are taken up in subsection 4.2. These issues of idealization and generality are questions about the structure of control at

\footnotetext{
26“Since two parabolas have three common tangents, the problem admits three solutions, including certain real solutions." (Beloch 1934, p. 187). 
play in the folding practice. What provides this structure of control of this practice and how is it regulated? At this point, we have met the major players who practised folding mathematically, know about their motivations and works and have convinced ourselves that folding can be reasonably called a practice. Our next step is to investigate the nature of the Rouseian situation of the mathematicians who practise folding. Or, to put it into Manders' words, what provides the structure of control in the folding practice?

A preliminary naive answer to our leading question already covers much ground: the mathematicians are in the situation that they are folding paper $^{27}$ to construct lines. The physical realities of paper restrict and justify how lines can be constructed by folding. To put it in a slogan: if you can fold it, then you can construct it. ${ }^{28}$ Because these constructions play an epistemic role in proofs by folding, the physical realities of paper control the permissible inferential actions in proofs by folding. And that is to say that the physical realities of paper provide a controlling structure in Manders' sense. Similarly, the mathematicians share the situation brought about by the physical realities of paper when folded. Thus, the physical realities of the material used justify and control the permissible inferential actions in arguments by folding.

This naive answer covers much ground because it correctly highlights the controlling effects of the material. The allowed moves - such as folding a point on a given line or folding two different points on two different lines - are not given in axiomatic form (even though they were later axiomatized), ${ }^{29}$ but are permissible because they can be performed on paper. This means that the permissible moves of reasoning by paper folding can be discovered by handling the material - Beloch discovered the Beloch-fold. This is quite different from the propositionalsymbolic geometric practices in which the allowed constructive moves are given by axioms or propositions before any reasoning is carried out; more on this in the section 6 of this article.

This points also to a similarity between a different diagrammatic practice and paper folding. Knot diagrams can visualize motion or constructions; (Giaquinto 2007, p. 263). De Toffoli and Giardino present knot diagrams as dynamic due, for example, to the visualization of the possible Reidemeister moves, indicating that the diagrams involve "visualizing motion"; (De Toffoli and Giardino 2014, pp. 839-841). Paper folding can also be thought as "visualized motion" since all the basic operations of folding point towards the possibility of visualizing them in the mind, rather than performing them in a haptic manner. However, this haptic quality is present in the proofs by folding as they appear in the practice; see e.g. our discussion of Row above. We will argue that this haptic dimension of manipulating a physical sheet of paper adds to the epistemic force of proofs by folding by providing correctives for errors of visualization.

The above answer to the question of what provides a structure of control for the folding practice is naive because it is simply not true that all constructive moves in folding are justified solely by the physical realities of paper. First, although lines are created as creases, points are sometimes regarded as resulting from intersection points of two creases, ${ }^{30}$ but may also be considered as basic objects, which are already given, i.e. not created from any basic folding

\footnotetext{
27 For ease of exposition, we will assume that the mathematicians fold paper. They could fold any other material that behaves similar to paper when folded, such as papyrus, tin-foil, etc.

${ }^{28}$ Notice the difference to Euclidean practice where not all drawings on paper are permissible constructions. Examples of non-permissible drawing in Euclidean practice include free-hand drawings, moving ruler constructions, Nicomedes chonoid etc. (see (Bos 2001) for a discussion of some of these).

29 With Justin's and Huzita's works.

30 "The point of intersection of the diagonals is called the center of the square" (Row 1893, p. 3)
} 
operation. ${ }^{31}$ Second, recall here Beloch who argued at length that a certain fold can generally be performed. It is true that if you cannot fold something in principle, then the move is not allowed in the folding reasoning practice. However, if one can fold a certain crease in a particular case, what guarantees that such a move can always be made? Our naive answer overlooks these questions of generality.

Our naive answer also overlooks the issues of idealization we drew attention to when presenting Row's folding of the geometric series. We will deal with this issue first, because it can be dealt with quickly, and then move on to the more serious worries about generalization. This will make the structure that provides control in the folding practice visible. We then move to questions about the epistemic strength of this structure.

\subsection{Idealization}

When reasoning by folding, just as in diagrammatic reasoning, we never run out of space. This suggests that the physical realities of paper provide a structure for control, not the physical reality of the actual sheet of paper used. To see this, let us consider the example presented in the introduction. We know by now that given a line and a point on a paper, we can fold a perpendicular to the line that hits the point. But can we always do this? Take an A4 sheet of paper and make a tiny fold that cuts across the bottom right corner. Now consider a point in the bottom left corner. The perpendicular to the line that hits this point lies largely outside of the paper. The physical realities of sheets of paper are such that they are finite in length. In what sense can we then still fold the perpendicular in this case? Are we not constrained by these physical realities in such a way that we simply cannot? The mathematicians have ignored this problem. The implicit assumption is that would we have folded 'more smartly' or used a larger sheet of paper (or both), we could have made it so that we can fold the desired perpendicular. Just as in Euclidean diagrammatic reasoning, we never run out of space when reasoning by paper folding.

We saw a similar kind of idealization with Row's infinite series. Row did not concern himself with what is physically possible. He allowed folding to be abstract even though he aims to "forceably impress the truth" on our minds. What matters, we suggest, are not the limitations of any given sheet of paper but rather what fold the physical realities of paper permit. By this we mean that when what limits the performability of certain folding constructions are certain details about the particular, physical sheet of paper that is used, then one can ignore these limitations; examples include the size of the sheet, its shape and thickness. When reasoning by folding, sheets of paper are always sufficiently large, the folding diagram is always suitably arranged, the paper can be folded multiple times and so on. Looking at the history of the practice, these limitations that arise from the physical realities of a particular sheet of paper were ignored by the mathematicians. As we will draw out in the next subsection, the limitations that arise from the physical realities of paper (rather than a sheet of paper) on the other hand play a fundamental role for folding as a reasoning practice.

\footnotetext{
${ }^{31}$ See for example section 4.2, for Justin's list of the seven basic operations. One may claim that new points can be marked as a result of basic operations, but these new points result from folding already given points. Note that (new) points can be obtained as a result of folding given points onto a new location (e.g. folding a point on a line, thus obtaining a point on a line); they can be also obtained as a "point of a crease"; for example: 13 halving the segment $\mathrm{AB}$ by folding $\mathrm{A}$ onto $\mathrm{B}$ gives you a point $\mathrm{C}$ in the middle of $\mathrm{AB}$.
} 
We argue that folding is a material reasoning practice. The above points about idealization reveal that, despite its material nature, reasoning by folding requires some, albeit limited, abstractions. This insight helps to clarify a possible misunderstanding. When we call reasoning by folding material, we do not mean that the reasoning can always be performed on the material; lines and points can "fall off" a given sheet of paper. Rather, as mentioned, we mean that the epistemic force of reasoning by folding stems from the material. It is part of the aim of the following sections to draw out what we mean by this.

\subsection{Permissible folding actions and their generality}

We saw already that Beloch was much concerned with the question whether a certain fold could always be carried out. She was not alone in this. Huzita and Scimemi give a list of what they conceive of as a "set of fundamental operations ... [which] will be universally accepted"; (Huzita and Scimeni 1989, p. 216). Of particular interest to us is the following operation:

(*) given a line $\mathrm{L}$, a fixed point $\mathrm{F}$ and another point $\mathrm{P}$. Fold such that $\mathrm{F}$ stays in place and $\mathrm{P}$ ends up on L. (Huzita and Scimeni 1989, p. 216)

To see whether this operation is indeed a general one, i.e. can be performed for any configuration of two points and a line, the reader should try this herself. Fix two points, $\mathrm{F}$ and $\mathrm{P}$, and a line $\mathrm{L}$ close enough to $\mathrm{P}$. Now fold such that $\mathrm{P}$ ends up on $\mathrm{L}$ and the fold runs through F. This is usually fairly easy to do. However, arrange the points so that $\mathrm{L}$ does not cut the circle around $\mathrm{F}$ with radius $|\mathrm{FP}|$. Folds that run through $\mathrm{F}$ can put $\mathrm{P}$ anywhere on this circle, but since $\mathrm{L}$ does not cut this circle, it is impossible in this configuration to fold as required in operation (*). In their paper, Huzita and Scimemi (1989) do not notice this. We see here that one must clarify under which conditions a folding operation can indeed be generally performed.

Jacques Justin gave in his 1989 paper "Résolution par le pliage de l'équation du troisième degré et applications géométriques" a list of seven basic, general folding operations; by "basic operation" we mean an operation which cannot be reduced to any other sequences of folding operations and which can always be performed, assuming that the points and lines are given in what we will call "generic position" (with the possible exception of operation (*)). By this we mean an arrangement of points and lines with only minor caveats (e.g. "lines are not parallel", "points are not on lines" etc.). It will be helpful to work our way through the list of basic folding operations, asking our question why the operation can be carried out in general. ${ }^{32}$ For Justin, the $P$ s denote points, $D$ s denote lines and $\rightarrow$ denotes "ends up folded onto." The operations are as follows; (Justin 1989 [1986], p. 257):

1. $P \rightarrow P, P^{\prime} \rightarrow P^{\prime}$ (fold along a crease going over two points)

2. $P \rightarrow P^{\prime}$ (fold a point $\mathrm{P}$ on another point $\mathrm{P}^{\prime}$ )

3. $P \rightarrow P, D \rightarrow D$ (fold along a crease perpendicular to $\mathrm{D}$ that passes through $\mathrm{P}$ )

4. $P \rightarrow D, D^{\prime} \rightarrow D^{\prime}$ (folding $\mathrm{P}$ on $\mathrm{D}$ while folding $\mathrm{D}^{\prime}$ on itself)

5. $D \rightarrow D^{\prime}$ (fold a line $\mathrm{D}$ on another line $\mathrm{D}^{\prime}$ )

32 Note that Huzita, while also trying to find the fundamental operations of folding, gave in: (Huzita 1989, p. 144), a list of only 6 fundamental operations and noted that there may be more operations to be added to this list. 
6. $P \rightarrow D, P^{\prime} \rightarrow P^{\prime}$ (operation (*) as above)

7. $P \rightarrow D, P^{\prime} \rightarrow D^{\prime}$ (folding two different points on two different lines) ${ }^{33}$

Operation 1 connects to the Euclidean basic operation of drawing a line segment through any two given points. That this is always possible, no matter how the points are arranged, was postulated by Euclid as proposition 1. In the folding practice, this is presented as an evident truth about what can be folded.

That operation 2 can always be performed is also self-evident. Notice however what it does. By folding one point onto another we create a crease in the middle between the two points. Given the former crease, that connects the two points, we have thus created a second crease that is perpendicular to and halves the (imagined or folded) line segment connecting the two points. Euclid needed an argument to show that his basic operations allowed such constructions. Indeed, it is, prior to its proof, not evident that ruler and compass constructions could construct such a line segment - this is why Euclid had to prove it. That is, unlike operation 1, which mirrored a basic Euclidean construction, operation 2 does not mirror a basic Euclidean construction.

We used the self-evidence of the general performability of operation 3 to illustrate how one can do geometry by paper folding in our introduction. Like operation 2, operation 3 is not mirrored by the three basic Euclidean constructions.

Operation 4 is not self-evident. First off, we are asked to fold such that the line D' ends up on itself. Similar to operation 3 we may think of this as folding D' onto itself, thus creating a crease perpendicular to it. However, it seems also possible to crease the paper along D' as this does not move the line. In fact, in some cases for operation 4, this is precisely how one must fold in order to perform the action described by operation 4 . This leads to a second point about operation 4: it cannot always be performed. Considering two parallel lines D and D' it is fairly easy to arrange the point $\mathrm{P}$ on the paper in such a way that $\mathrm{P}$ cannot be folded onto $\mathrm{D}$ in the desired way (when $\mathrm{D}$ and $\mathrm{D}^{\prime}$ are close to each other and $\mathrm{P}$ is far away from the lines for example). Justin notices this and demands in his list of operations that D and D' not be parallel. That is, he demands that they are in generic position. Given this constraint ${ }^{34}$ operation 4 can be seen as the projection of $\mathrm{P}$ onto $\mathrm{D}$ along D'. Because such a projection can always be performed (under the given constraints), operation 4 can always be performed.

That operation 5 can always be performed is again self-evident. In fact, as Justin mentions, if D and D' intersect, then there are two folds that fold D onto D', each of these folds bisecting two of the four angles at the intersection of $\mathrm{D}$ and $\mathrm{D}$ '.

Operation 6 was discussed above, and operation 7 is the Beloch fold. As we saw, that such a fold always exists was proven in 1934 by Beloch. However, to prove that such a crease always exists, Beloch showed that finding it is equivalent to finding a common tangent to two parabolas (cf. section 3 for elaboration). Since the number of common tangents to two parabolas is always greater or equal to 1 the crease always exists. Beloch thus shows that a certain manual folding operation can be carried out in general by relying on analytical algebraic reasoning.

We have seen that any line can be constructed in the crease-diagram that can be obtained from a single fold. "If you can fold it, then you can construct it" was the slogan. In this

\footnotetext{
33 Justin also gave in his paper a list of restrictions on these operations.

${ }^{34}$ Justin also adds that P not be on D to avoid trivialities; (Justin 1989, p. 257). 
subsection we showed that the mathematicians reasoning by paper folding were concerned with the question whether their folds could generally be performed; whether a particular case could be elevated to a basic folding operation. Such arguments for the general performability of the fold were usually performed in reasoning idioms other than folding; we discussed Beloch and her use of the resources of symbolic-propositional analytic geometry. To recall: the operation of folding three different points on three different lines can be also considered as a permissible operation, however, it is not generally practicable, because if the points and lines are in generic position, then there is no crease along which we can perform the fold; this is because it would be equivalent to finding a comment tangent to three parabolas in generic position. However, just by trying (unsuccessfully) to fold three given (generic) points on three lines, one cannot prove that this operation in fact cannot be performed in general. This shows that one aspect of the work done on paper folding is to investigate the generality of the permissible constructive moves. These are not given to us at the outset. Rather, the generality of the permissible constructive, material moves is discovered and proved. With the (re)discovery of the Beloch fold by Huzita and Justin, there seemed to be no guarantee that there are no other basic folds that can always be performed but simply have not yet been found. Indeed, Huzita and Scimemi note in regard to a list of five fundamental operations they give that it should "be noticed [that] those [operations] are obviously not listed [sic.] all the possible folding operations" (Huzita and Scimeni 1989, p. 216). The proof that the above list of the seven operations is complete was published only in 2009 by Roger Alperin and Robert Lang; (Alperin and Lang 2009, pp. 374377).

\section{Permissible inferences}

In the last section we showed that several basic folding operations are not obviously always performable. Beloch's work in particular dealt with the question of whether her fold can generally be performed. This question is the heart of one reasoning-tradition in the folding practice. We will now turn our attention to another reasoning-tradition of this practice: proving theorems (such as Row's proof of the value of the geometric series) and constructing shapes (such as Row's envelop of a parabola) via paper folding.

The epistemic force of folding proofs has been differently judged. On the one hand, the mathematician Row held that folding can "impress the truth" of geometrical propositions "forcibly on our minds". The philosopher Marcus Giaquinto, on the other hand, argues that proofs by folding can help us to form a belief, but folding is epistemically too weak to support such beliefs on its own. Our analysis of the epistemic force of proofs by folding as they appear in practice reveals that it is stronger than Giaquinto realizes, even though it may fall short of Row's hopes.

Row claimed that proofs by folding "impress the truth" of the theorems "forcibly on our minds". At least superficially, there is some truth to this claim. Proofs by folding have a seemingly inescapable immediacy. When we fold a square piece of paper along its diagonal, it seems obvious that the resulting two triangles are congruent, for example. ${ }^{35}$ On second thought, however, we realize that the edges of the paper do not perfectly align. Even if we folded very precisely, our physical world is messy and edges of a sheet of paper never align quite as nicely

\footnotetext{
${ }^{35}$ See e.g. (Row 1893, p. 5).
} 
as they do in abstract mathematical thinking. How secure then are arguments based on folding proofs? How rigorous are arguments by paper folding?

It is striking that, as far as the authors are aware, there is not a single folding-proof of a statement which turns out to be incorrect. If you can fold it, it seems, then other reasoning idioms will not disagree with the result, in the sense that the construction cannot be shown via other idioms - to be impossible. Notice that this is unlike diagrammatic reasoning, in which, to give the well-known example, the (incorrect) statement "every triangle is isosceles" can be "proven". The would-be proof relies on drawing the intersection of certain lines to lie within the triangle even though they should, had one "drawn better", lie outside. This mistake in the inside-outside relation plays a fundamental role in the "proof" of the incorrect statement. Row (1893) points to this "fallacy" (p. ix) to motivate geometry by paper folding. As he correctly points out, such an incorrect proof cannot be given via folding. When folding this "proof" 36 the lines will always meet outside of the triangle. One cannot "draw badly" when "proving" (i.e. attempting to construct) via folding in the same way as one can in diagrammatic proofs.

The above highlights some of the epistemic strengths of proofs and constructions by folding: they are convincing and seem, at least for now, irrefutable. But it does not deal with the issue of our messy world. Some basic folding operations ${ }^{37}$ ask us to align creases in the paper. Because our world is messy, we can never perfectly align these creases. How trustworthy are arguments based on such imperfections?

According to Gianquinto (2007), the folded paper alone is not sufficient to discover a belief. ${ }^{38}$ Giaquinto reminds us of the doubt we may have when reasoning on sense experience alone. When wondering whether a table fits into a room without the possibility to measure, we may think that it fits but are ready to admit that we may be wrong. This feeling that we might be wrong is Giaquinto's clue that there is more involved in proofs by folding than pure sense experience. In practice, mathematicians are indeed not relying solely on the sense experience obtained from a fold. The folding-proofs Row gave, for example, are not reliant purely on the sense experience of the folded paper. They are accompanied by text, which provides instructions and guides the reader's focus. As Stone and Stojnic (2015) highlight, such guidance of focus may be necessary for folding proofs to function properly. ${ }^{39}$

Giaquinto favors an epistemological account, which draws on the concept of cognitive states. Folding becomes visualizing in the mind rather than the perception and handling of a piece of paper; (Giaquinto 2007, p. 60). ${ }^{40}$ As Giaquinto correctly points out, beliefs acquired by such visualizations of folding may not be reliable. For example, one may fail to correctly visualize what one intended to visualize. Such problems do not arise when reasoning as the mathematicians do when they actually fold paper. We cannot fail to visualize what we intended

\footnotetext{
${ }^{36}$ Or perhaps: a folding-version of this proof.

${ }^{37}$ For example, operation 5 discussed in section 4.2 .

${ }^{38}$ e.g. "I will argue that those empirical routes to the belief are not ways of discovering it" (Giaquinto 2007, p. 52).

39 "If [folding] demonstrations do have precise content, it can't just follow from the physical effects of the demonstrations alone; there must be other cues that determine the content of the speaker's argument. These cues must help viewers track the features of the demonstration that matter, and help viewers not be distracted by features of the demonstration that don't." (Stone and Stojnic 2015, pp. 81-82)

${ }^{40}$ As we have seen, this is not how the mathematicians who fold paper reason, i.e. Giaquinto is losing traction with actual practice here. Arguably Giaquinto was not interested in folding as a practice but rather in folding as an epistemic activity.
} 
to visualize when folding paper. The action of folding paper together with the resulting creasediagram does the visualizing for us.

In particular, Giaquinto did not aim at analyzing folding as a reasoning practice. We now return to our analysis of this practice, and return later to Giaquinto's arguments regarding the rigor of reasoning by paper folding.

In order to deal with folding as reasoning practice, recall that, alignment of lines by folding is always imperfect. Euclidean diagrammatic practice faces similar problems. Lines in Euclidean diagrams have widths; certain aspects of these diagrams are unstable under perturbation; circles are imperfectly drawn etc. That Euclidean diagrammatic reasoning is nonetheless rigorous has been successfully argued by Manders (2008). To recall, a fundamental insight of Manders is that not all but only some information may be read off diagrams in Euclidean reasoning practice. Manders divides the attributes of a diagram into two kinds: exact and co-exact. The exact attributes of a diagram are unstable under perturbation. Examples include the lengths of line segments or the width of angles. This kind of information is not read off the diagram in Euclidean practice. Co-exact attributes are stable under the slight deformations that appear when re-drawing the diagram. Examples include inside-outside relations or recognition of regions. Information of this kind is read off diagrams in Euclidean practice and relied upon in (textual) argument. The diagram thus stands to license certain arguments, and this makes the constructive moves for drawing Euclidean diagrams inferential actions.

Manders' work is a reconstruction of Euclidean practice, successfully showing that this practice was rigorous. Note that the claim is not that the practitioners of Euclidean practice thought in terms of exact and co-exact attributes; there is no mention of such terms in Euclid's works. Rather, Mander's terminology provides a philosophical framework in which the rigor of Euclidean practice can be understood. We aim to do the same for the folding practice in this section.

Giaquinto (2007) asks us to imagine a square piece of paper whose corners we fold to its center (p. 51). It is instructive here to read Giaquinto in full, including the figure he draws (see figure 4):

"Imagine a square. Each of its four sides has a midpoint. Now visualize the square whose cornerpoints coincide with these four midpoints. If you visualize the original square with a horizontal base, the new square should seem to be tilted, standing on one of its corners, "like a diamond" some people say. [...] Clearly, the original square is bigger than the tilted square contained within it. How much bigger? By means of visual imagination plus some simple reasoning one can find the answer very quickly.

By visualizing this figure, it should be clear that the original square is composed precisely of the tilted square plus four corner triangles, each side of the tilted square being the base of a corner triangle. One can now visualize the corner triangles folding over, with creases along the sides of the tilted square." 


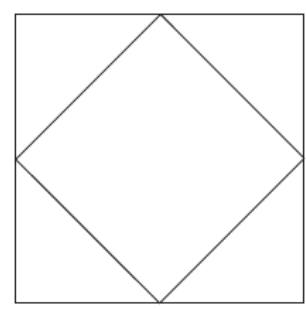

Fig. 4: Giaquinto's square (ibid, p. 51).

As Giaquinto states "Many people conclude that the corner triangles can be arranged to cover the tilted inner square exactly, without any gap or overlap" (ibid.). Such people might be led to deduce, so Giaquinto reasons, that the sum of the area of the four triangles is exactly half of the area of the square piece of paper we started with. In fact, a symbolic-propositional statement of this can be proven. Giaquinto asks us how, from our folded piece of paper, we can be certain that the four triangles completely cover the remaining square.

Giaquinto raises (amongst others) a worry about equality of size here. Equality of sizes are exact attributes of a diagram and thus is not a piece of information that can be read off the diagram in Euclidean practice. However, proofs by folding consist of more than some text and the crease-diagram, as they also involve the haptic motion of folding. We will argue that this haptic moving of the two-dimensional plane through three-dimensional space changes what counts as exact and co-exact attributes in the folding idiom with respect to the Euclideandiagrammatic idiom.

Notice here also that, despite superficial similarities, Giaquinto's fold is different from the first step of Row's proof of the geometric series discussed in section 3.1. Row fully triangulated the square by first folding one corner onto the opposite corner, then folding the other two corners onto each other, then halving the piece of paper vertically, then halving it horizontally and so on. This establishes the mid-points of sides of the relevant squares. Furthermore, Row opens the paper up again after a fold. Row reasons on the crease-diagram (infused with what can be learned from the folding motion, see below). Giaquinto does not reason like this. He takes the mid-points of the edges of the (bigger) square as given and then folds four triangles simultaneously. He aims to reason on the already folded piece of paper, not on the crease-diagram.

Row states that folding one corner onto the opposite corner results in two congruent triangles; (Row 1893, p. 5). At least on the face of it, Row seems to be reading off an equality of size from his folding here. Although Row seems to lack Giaquinto's awareness of the philosophical issues at play here, the reasoning Row follows suggests that we should inquire more deeply what can be inferred from the various folding operations. We now turn to do so in the next subsections.

\subsection{What can be read off the folding operations?}

Let us then go through the basic folding operations and ask what kind of information can be rationally obtained by performing the operation. We then come back to the question whether Row was justified in stating that the resulting two triangles are congruent. 
Before we go through the basic folding operations, it is important to realize that the mathematicians idealize while reasoning by paper folding (cf. section 3.1). In particular, when they fold a point onto a point, a line onto a line, a line through a point or a point onto a line, these actions are always taken to be successfully carried out with absolute mathematical precision. Giaquintonian worries that certain lines (or points) may not align properly are ignored. ${ }^{41}$ We call this "controlled idealization". We can only infer perfect alignment of two geometric objects if they were the target of our folding operation. If, when folding a point onto a point, two lines seem to perfectly align, we cannot infer from folding alone that these lines are, in fact, perfectly aligned. Such an idealization would be an instance of uncontrolled idealization. Uncontrolled idealization is what creates the difficulties with Row's fold discussed below. What follows will make more precise what we mean by controlled and uncontrolled idealization. To do this, we first establish a sense of what is meant by "controlled idealization" and then highlight instances of uncontrolled idealization.

Basic folding operation 1 is to fold a crease between the given points $\mathrm{P}$ and $\mathrm{P}$ '. On the resulting crease-diagram there is a crease with $\mathrm{P}$ and $\mathrm{P}^{\prime}$ on the line created by the crease. We can now read off from the crease diagram that $\mathrm{P}$ and $\mathrm{P}^{\prime}$ are indeed on this line. This is simply an instance of controlled idealization.

Basic folding operation 2 is to fold a point $\mathrm{P}$ onto a point $\mathrm{P}$ '. The resulting creasediagram has two points and a crease in between these two points on it. From the diagram alone, we cannot infer that the crease created is a line that is equidistant from $\mathrm{P}$ and $\mathrm{P}$. ${ }^{42}$ However, to align the two points we folded the paper. By carrying out this action we can see that the line is indeed equidistant from $\mathrm{P}$ and $\mathrm{P}$ ' because the paper between the crease and $\mathrm{P}$ is covered by the paper between the crease and $\mathrm{P}^{\prime}$. We see here that reasoning by paper folding relies not solely on the crease-diagram; the haptic motion of folding paper informs the rigor of the reasoning. When folding one thus needs to think of the crease-diagram and the folding together when considering the reliability of this form of reasoning. The kind of reliable information that is obtained by folding lies thus in both the crease-diagram and the haptic motion of folding. And this shows a difference of co-exactness between diagrammatic reasoning and reasoning by folding. In diagrammatic reasoning, co-exact attributes provide reliable information that can be read off the diagram. In folding, reliable information stems not only from the diagram alone but is also informed by haptic motion. Co-exact attributes of a fold are thus not (necessarily) ${ }^{43}$ found solely in the crease-diagram but latch onto the crease-diagram and haptic motion tuple.

In basic folding operation 3 one folds a line D onto itself such that the resulting crease passes through a given point $\mathrm{P}$. From the resulting crease-diagram alone we cannot infer that the newly created line is perpendicular to D, because, as Manders has correctly pointed out, in a purely diagrammatic idiom the size of an angle is an exact attribute of the diagram. Again, the haptic motion of folding adds information here. When folding a line onto itself, controlled idealization allows us to infer that the line perfectly aligns with itself. Furthermore, a crease is always at a right angle to the line (or lines) along which it was folded since the haptic motion shows that one places an angle on an equally sized angle, such that their sum is $180^{\circ}$. Thus, the angle between $\mathrm{D}$ and the resulting crease are right.

\footnotetext{
41 "Is it not the same when through visualizing one concludes that the four corner triangles of a square would fit exactly onto he tilted inner square without overlap?" (Giaquinto 2007, p. 53)

${ }^{42}$ Because distances are exact attributes of a diagram; cf. (Manders 2008).

${ }^{43}$ The co-exact attributes of basic folding operation 1 rely solely on the diagram.
} 
Notice here that there is an epistemic interplay between the folding operations. Operation 1 allows us to create a line between the points $\mathrm{P}$ and $\mathrm{P}^{\prime}$. Operation 2 halves this line. From operation 3 we can infer that the point which lies at the intersection between the two creases created earlier is the midpoint between $\mathrm{P}$ and $\mathrm{P}$ '.

In basic folding operation 4 one folds a point $\mathrm{P}$ onto a line $\mathrm{D}$ whilst not moving a given line D'. As mentioned in section 4.2, this fold can in general only be performed when the two lines and the point are given in generic position. This operation marks a point $\mathrm{P}^{\prime}$ on line $\mathrm{D}$ which stands in a specific relation to $\mathrm{P}$. This piece of information can then be used in further argument.

Given two lines, D and D', basic folding operation 5 tells us that we can fold D onto D'. When given in generic position, D and D' intersect. In such a case, the newly created crease C divides two opposite angles formed by D and D'. The size of the angle between $\mathrm{C}$ and D (or D') is a piece of exact information in a purely diagrammatic reasoning idiom. In the folding idiom, from the haptic motion performed when folding it can be read off that the angles between $\mathrm{C}$ and $\mathrm{D}$ and between $\mathrm{C}$ and D' are the same. Thus, $\mathrm{C}$ is the angle bisector of (two of the angles between) D and D'.

Basic folding operation 6 starts with two points, $\mathrm{P}$ and P', and a line D. One folds through $\mathrm{P}^{\prime}$ such that $\mathrm{P}$ ends up on $\mathrm{D}$. In section 4.2 we pointed out that this fold can only be performed in general when $\mathrm{P}, \mathrm{P}$ ' and $\mathrm{D}$ are given in generic position, i.e. when $\mathrm{P}$ is "close enough" to the line D (see section 4.2). As in operation 4, this marks a new point on the line D.

Basic folding operation 7 is the Beloch fold, i.e. one folds $\mathrm{P}$ on line $\mathrm{D}$ and point $\mathrm{P}$ ' on line D' simultaneously. By folding $\mathrm{P}$ onto $\mathrm{D}$, we mark a point $\mathrm{p}$ on $\mathrm{D}$ where $\mathrm{P}$ is aligned during the folding action with D. Similarly, a point p' is marked on D'. Now imagine (or fold) a line L between $P$ and $p$ and a line L' between P' and p'. From basic folding operation 2 and 3 we know that the Beloch-crease is perpendicular to both $\mathrm{L}$ and $\mathrm{L}^{\prime}$ and halves these two lines. This is precisely the information needed from the fold to double the cube as in section 2 . Notice that to argue that the constructed line-segment is of the desired length, one needs to apply propositional-syntactic reasoning idioms (as presented in section 2; see footnote 17). The point here is that the Beloch fold provides the kind of information that is necessary for a propositional-syntactic reasoning to successfully argue that the length of the line-segment is the cube root of 2.

We now return to the question of the congruence of triangles. Recall that Row folded a square piece of paper along its diagonal and claimed that the two resulting triangles are congruent. Call this "Row's fold". Has he obtained this information solely from the fold and the resulting crease-diagram?

There are at least three ways in which Row's fold can the thought. To get at these, let us consider a square whose corners are ABCD. One way to think of this fold is to fold the crease passing through points $\mathrm{B}$ and $\mathrm{D}$ as in operation 1. To assume that this fold aligns points $\mathrm{A}$ and $\mathrm{C}$ would be an instance of uncontrolled idealization as such inferences are not supported by the folding operations as discussed above. Such an inference is thus not permissible. This way of thinking about Row's fold is not particularly helpful.

Perhaps it is more natural to think of Row's fold as aligning points A and C. Alas, this is also not helpful as such a fold does not allow us to infer that the lines $\mathrm{AB}$ and $\mathrm{BC}$ align on pain of uncontrolled idealization. 
The most suitable way of thinking about Row's fold is to think of it as aligning two lines as in operation 5. This is in fact how Row reasoned, when constructing his square (see section 3.1). Say we fold line AB onto line BC. Since we know that the line AB and BC are equal in length (they are sides of a square), we know that $\mathrm{A}$ and $\mathrm{C}$ perfectly align by performing this fold. Furthermore, by aligning $\mathrm{AB}$ and $\mathrm{BC}$, we know that the resulting crease halves the angle $\mathrm{ABC}$. Since the angle is right, the resulting angles are 45 degrees. Note that Row constructs his square (as indicated in section 3.1) in such a way such that the crease ends at a point, which is only then denoted as the point $\mathrm{D}$. That is, the vertex $\mathrm{D}$ is constructed as the end-point of the crease-segment, hence the crease segment is constructed as the diagonal of the square. This enables Row to read off from the folding diagram the properties of a diagonal, in contrast to Giaquinto's treatment. Furthermore, we know that the angles DAB and BCD remain right because they are not interfered with by the folding operation. Thus, we know that the two triangles have two angles which are equal (DAB and BCD) and the line between these two angles ( $\mathrm{AB}$ and $\mathrm{BC}$ ) are equal in length. We can thus argue by the side-angle-side postulate that the two triangles are congruent. Indeed, this seems necessary because inferring from the fold alone that the lines BC and DA align perfectly would be an instance of uncontrolled idealization.

Whilst this resolves the question why the two triangles are congruent, it does so by relying on a postulate about the congruence of triangles. This postulate was not shown by Row as what can be read off the haptic folding operations, and we confess our inability to see how it could ever be proved by a reasoning idiom that relies purely on folding (and text). Row may thus have been a bit too hopeful when he claimed that when doing geometry by paper folding "it would not be necessary to take any statement on trust"; (Row 1893, p. ii). Our philosophical analysis of the rigor of reasoning by paper folding suggests that it is indeed necessary to accept certain statements on trust. ${ }^{44}$ This adds to the point we made earlier about different reasoning idioms being used in the folding practice.

We have seen earlier that the mathematicians practising folding switch with ease between symbolic-propositional reasoning idioms and a folding idiom. In this section, we pointed out that the folding idiom relies not only on text and the folding diagram but also on the haptic motion of the fold. Because this haptic motion allows for controlled idealizations, it provides information beyond empirical evidence. Folding as practised is thus more rigorous than Giaquinto's points suggest ${ }^{45}$, which we showed by highlighting what pieces of information can be rationally obtained from the basic folding operations. This rigor needs to be augmented by some "statements taken on trust" for certain arguments to function properly.

\section{Folding as a material reasoning practice}

As we saw in section 2, Row wrote

"This would give [the children] neat and accurate figures, and impress the truth of the propositions forcibly on their minds. It would not be necessary to take any statement on trust."

\footnotetext{
44 This is a similarity to Euclidean geometric reasoning.

${ }^{45}$ However, recall that Giaquinto may not have been interested in the rigor of this reasoning practice.
} 
This quotation shows that for Row, one does not have to justify folding in terms of other idioms (propositional, symbolical, etc.). Beloch however acknowledged folding as not necessarily evidential. We would like to emphasize that for different mathematicians, folding practice had different roles. But both Row and Beloch pointed to the epistemic force of reasoning by paper folding. Our claim, as was already indicated in earlier sections, is that this force is obtained from the physical realities of the material used. It is in this sense that we call reasoning by paper folding a material reasoning practice.

In Euclidean diagrammatic reasoning the constructive moves in a diagram are inferential actions. ${ }^{46}$ As Larvor writes, "[the constructive moves] are strictly controlled by the first three postulates - nothing else is permitted". ${ }^{47}$ But there were debates about whether other constructive moves should be permitted. There is a host of marked and moving ruler constructions, which have been discussed since the time of the ancient Greeks. ${ }^{48}$ The use of such tools and instruments was not well accepted as truly geometrical by the mathematical community at large; indeed, according to Plutarch,

\begin{abstract}
"Plato himself reproached Eudoxus and Archytas and Menaechmus for setting out to remove the problem of doubling the cube into the realm of instruments and mechanical devices, as if they were trying to find two mean proportionals not by the use of reason but in whatever way would work. In this way, he thought, the advantage of geometry was dissipated and destroyed, since it slipped back into the realm of senseperception instead of soaring upward and laying hold of the eternal and immaterial images [...]" (Plutarch 1961, pp. 121-123)
\end{abstract}

Folding is also an inferential action. This is because we start with some condition (e.g. "given a square"), realise it on the sheet of paper (fold a square as Row did), then construct lines by folding (e.g. a diagonal of the square) and at the end we have some more information which we can read off our sheet of paper. Unlike Euclidean diagrammatic constructions however, the constructive moves of folding are not justified by the tools we use. Reasoning by paper folding does not rely on tools such as rulers or compasses. Rather, a constructive move is justified just in case it can be carried out on the sheet of paper. The (idealized) physical realities of paper thus control the constructive moves. Because such moves are inferential actions, the physical realities of paper control the inferential actions of reasoning by paper folding.

Not all diagrammatic reasoning relies on tools or delineating all permitted constructive moves for diagram construction at the outset. As an example, consider diagram construction in diagrammatic reasoning in knot theory:

"In order to draw a diagram for a knot, we project it on a plane, by keeping track, for each crossing in the projection, of which arc goes over and which under. Not all projection directions are allowed, since the projection must be regular, i.e. (i) the

\footnotetext{
${ }^{46}$ See (Manders 2008) and (Larvor 2017).

${ }^{47}$ In footnote 13, Larvor adds: "With the exception of Elements I.4, where a triangle is rigidly displaced. This is well-known to commentators" (p. 7).

${ }^{48}$ Many of these can be found in (Bos 2001) 
intersection points are in finite number, (ii) they are transversal and (iii) no more than two arcs meet at a time." (De Toffoli and Giardino 2013, p. 832)

In this case diagrams are projections of three-dimensional objects, which have possibly been subject to "manipulative imagination" (p. 829). These objects - knotted pieces of string - are subject to the physical realities of string. There is a similarity to reasoning by paper folding.

By folding paper, we obtain a piece of paper with lines scattered across it: the creasediagram. We nonetheless argue that folding is not a diagrammatic reasoning practice. Unlike Euclidean diagrammatic reasoning, the permissible constructive moves of folding are not explicitly given at the outset in propositional form. In fact, there is a reasoning tradition which explores what the permissible constructive moves of folding are; cf. section 5. In the folding practice there is also no debate about whether other tools for construction should be permitted. The crease-diagram is neither a representation of what is reasoned about as are the diagrams of knot theory, nor does folding require "manipulative imagination". Indeed, as noted above, we can imagine how folding creates a fold, but we cannot manipulate it as we want, creasing, for example, just near the desired place. Also, as mentioned above, the idealization that takes place with folding is a controlled one, which is also true for knot-diagrams. However, in contrast to knot-diagrams, reasoning by folding is more direct than diagrammatic reasoning, as it does not come to represent a line (or a knot), it creates one. This points to another aspect we mentioned above, that the haptic motion must be taken into account when considering this reasoning.

Larvor (2017) argues that there is an essential use of non-textual inferential actions in diagrammatic reasoning. To translate diagrammatic reasoning into other idioms is to change the object on which the inferential action is carried out: traduttore traditore. In the case of folding, such translations betray the original reasoning less. This is because, unlike in Euclidean practice, in the practice of folding as depicted in this article, many practitioners have modern formalised notation at their disposal. Some of the works on folding discussed in this article move with ease from the haptic-motion part of the folding idiom to a modern propositional one and back again. ${ }^{49}$ Some scholars seem to prefer haptic motions; Row included very few mathematical arguments given in a propositional idiom. Others, such as Beloch, argued entirely within the propositional idiom; there are no pictures of any kind in Beloch's work on folding and to prove the generality of her fold, she needed to turn to an argument form analytic geometry. The situation which the practitioners of folding share (Rouse) is one in which different idioms not only exist simultaneously - this is true of Euclidean practice as well - but in which the idioms are readily interchangeable and are regularly interchanged. Diagrammatic reasoning stops being diagrammatic reasoning when all the diagrams are translated into propositional idioms. This is not true of folding. A proof by paper folding does not actually have to be folded (even though it could be). Traduttore partecipante may be the better slogan here.

To conclude: to construct a line, a bisector, or marking new points is part of the information that can be read off the various folding actions. The inferential actions of folding (the folding operations) are justified by the physical realities of paper. The epistemic force of these inferential actions is harvested both through propositional and through folding-

\footnotetext{
${ }^{49}$ For an insightful account of conceptual agility in modern mathematics, see Manders' unpublished article "Euclid or Descartes? Representation and Responsiveness".
} 
diagrammatic idioms, where it either functions as what provides evidence for the proposed claim, or only functions as "an a priori means of acquiring belief" (Giaquinto 2007, p. 67). The practice of mathematical paper folding is thus neither purely propositional nor diagrammatic in the sense used by Manders, Larvor, De Toffoli and Giardino. We propose to name this reasoning by the source of its epistemic force: the material.

\section{Enabling a mathematical culture of folding}

We have argued that folding is a material reasoning practice. We now turn our attention to the theme of this special issue: enabling mathematical cultures. We suggest that the mathematical practice of paper folding as performed and sustained by the above described community of mathematicians during the $20^{\text {th }}$ century, has enabled a mathematical culture of folding.

What do we mean by "enabling"? The term may be understood as indicating a space either virtual or physical - where mathematicians can meet and communicate. If paper enables a folding culture - we engage with this question below - then the way paper enables such a culture is not by providing a space for discussion. Rather, paper sanctions and disqualifies certain constructive moves. ${ }^{50}$ That is, as we showed, paper enables a certain kind of reasoning. (Paper as a material on which formulas and mathematical texts are written may also enable mathematical cultures, but this is not the topic of this article)

When thinking of (folded) paper as a medium that enables modes of reasoning, we may think that in doing so it establishes certain customs, beliefs and knowledge. This connects nicely to Tylor's definition of culture of 1871 :

Culture ... is that complex whole which includes knowledge, belief, art, law, morals, custom and any other capabilities and habits acquired by man as a member of society (Tylor 1871, p. 1)

Tylor's definition is what Kroeber and Kluckhohn call "descriptive” (1952, p. 43). It enumerates what belongs to a culture. As Kroeber and Kluckhohn point out, such enumerations can never be exhaustive and what is not mentioned tends to be left out in further thinking. Besides such general criticisms, there is a more direct worry in connection to folding as a culture, which has to do with the idea of shared beliefs and customs. To get at this, let us consider cultures as conglomerations of shared beliefs.

The idea that cultures are conglomerations of shared beliefs has tradition. Banks and McGee Banks write:

Culture also consists of the shared beliefs, symbols and interpretations within a human group. ... The essence of a culture is not its artefacts, tools, or other tangible cultural elements but how the members of the group interpret, use, and perceive them. (Banks and McGee Banks 1989, p. 8)

More recently, the shared beliefs of a culture have been cashed out as mental states:

\footnotetext{
${ }^{50}$ It may be necessary to argue that such moves can be generally performed; see section 4 . 
Culture is information capable of affecting individuals' behavior that they acquire from other members of their species through teaching, imitation, and other forms of social transmission. By information we mean any kind of mental state, conscious or not, that is acquired or modified by social learning and affects behavior. We will use everyday words like idea, knowledge, belief, value, skill and attitude to describe this information. (Richerson and Boyd 2005, p. 5)

Culture as mental states is an extreme form of the culture-as-shared-beliefs idea because it pushes culture into (the skull of) a single agent. In Richerson and Boyd's definition, there is no need for a community to exist; a single agent could have all the right kind of mental states to carry an entire culture "in her head". This, we suggest, is an unreasonable understanding of the term "culture". We thus discard such radical forms of the culture-as-shared-beliefs idea and consider how more moderate versions of it align with the thought that folding may be a culture.

Folding can lead to shared beliefs and knowledge because folding can, with the caveats discussed earlier, prove mathematical theorems and construct mathematical objects. But neither folding nor paper enables these beliefs or knowledge. The mathematical theorems proven via folding were already known from other mathematical idioms; folding has not led to any new theorems (yet?). What paper enables is not the belief in a mathematical proposition but the reasoning that supports this proposition. The question is thus whether the mathematicians who fold paper share beliefs about this type of reasoning.

The answer to our question is negative. As highlighted in section 2, mathematicians folded paper for various reasons. They had different backgrounds in terms of the time and place they lived in. Row was influenced indirectly by Fröbel's activities and their reconceptualization in India. He was interested in mathematical education and attempted to popularize folding as a geometrical practice on equal footing with the axiomatic approach. Hurwitz saw paper folding mainly as suitable for constructing regular polygons, but did not consider his results important enough to be published. Beloch treated folding as a piece of research mathematics but also thought of it as having pedagogical value. She published only few articles on paper folding. In contrast to Row, Beloch was a professor at a university, and her interests were mainly in other fields (algebraic geometry, topology and starting from the 1930s, also photogrammetry). Huzita had a very different aim and belief regarding the relations between folding and mathematics; he was one of the first mathematicians who aimed at a full axiomatization of folding based geometry. There is thus no shared belief on the value of this type of reasoning.

Furthermore, mathematicians disagree on the epistemic strength of reasoning by paper folding. We highlighted that Row attributed rigor to proofs by folding. He was not alone in this; cf. (Friedman 2018, section 5.1.1). Others disagreed; for example, Young and Young's (1905) critical view on Row's work.

It is also unclear to what extent Beloch, Justin and Huzita regarded proofs by folding as rigorous. Justin and Huzita transposed folding proofs into a symbolic propositional idiom. To conclude that they were hence doubtful about the rigor of proofs by folding would, however, be misguided. Formalizing the basic folding operations in the 
way they did allowed them to access questions about what folds can generally be performed. We have highlighted that this is one tradition (the other being proving theorems and constructing shapes) we can find in the folding practice; cf. section 5.

Focusing again on shared beliefs, Netz highlights: "Shared beliefs are much less common than shared practices ... Beliefs cannot explain the scientific process" (1999, p. 2) Netz understands "practices" here as way of doing things. This is useful because it allows for cohesion within a group, even if there is disagreement about certain beliefs. This also connects with Rouse's point about practices being shared situations rather than systems of beliefs.

Kroeber and Kluckhohn (1952, pp. 50-55) have pointed out that normative definitions of culture with an emphasis on rules or ways of doing things - and we may read Netz' quotation as such - stress the idea of artificiality and arbitrariness of cultures. ${ }^{51}$ This argument is a point of discontinuity with a Rousian understanding of paper folding as a shared situation. Reasoning by paper folding, we argued, draws much of its strengths from the physical realities of the material folded. These physical realities provide a mechanism of control for the norms of reasoning by paper folding.

Kroeber and Kluckhohn provide a definition of culture of their own:

Culture consists of patterns, explicit and implicit, of and for behaviour acquired and transmitted by symbols, constituting the distinctive achievements of human groups, including their embodiments in artefacts; the essential core of culture consists of traditional (i.e. historically derived and selected) ideas and especially their attached values; culture systems may, on the one hand, be considered as products of action, and on the other as conditioning elements of further action (Kroeber and Kluckhohn 1952, p. 181)

Kroeber and Kluckhohn come to the culture term from a social scientific and specifically from an anthropological point of view. They think of the values that are attached to the traditional ideas that make up the essential core of culture as the "dos and don'ts" that shape social groups. One would be hard pressed to define a mathematical folding culture along these lines because the approaches to folding that were taken are too heterogeneous: some preferred fully syntactic-propositional idioms (e.g. Beloch), others were attracted by the haptic motions of folding (e.g. Row). This is not to say that the mathematicians who reasoned by paper folding did not share any values. Most, if not all, were working in the context of the rise of mathematical modernism, a movement that aimed to formalize entire mathematical domains; see (Mehrtens 1990). "The modernist ideology in mathematics preached renunciation from the world, in the sense that one did not do geometry or analysis [...] by taking as given what is presented by idealized common-sense" (Gray 2006, p. 390). Mathematical modernism influenced the folding practice and its values were shared by many (if not most) mathematicians who practised folding. Yet these values are too broadly shared amongst mathematicians to define a folding culture.

\footnotetext{
51 "The 'arbitrariness' of a cultural phenomenon is a function of its particular historical determination. 'Artificiality' is related to a different set of problems hinging on the role of culture in human life. Is it a thwarting or fulfilling or both? Is man's 'culturalness' just a thin film, an epiphenomenon, capping his naturalness? Or are cultural features in man's life so important that culture becomes the capstone to human personality?" (Kroeber and Kluckhohn 1952, 52) 
Mathematical cultures will be influenced by social norms such as Kroeber and Kluckhohn's dos and don'ts. Our focus here is another. We submit that the mathematicians discussed in this article share an appreciation for the kind of reasoning that emerges when proving by paper folding. That is, they value the Rousian situation they all find themselves in.

None of what we have said amounts to a definition of mathematical culture and we do not attempt to give such a definition here. Instead, we suggest to reflect on what has been said with an eye towards learning what mathematical cultures can be like. If we take for granted that mathematical paper folding is a mathematical culture - and we believe this to be a sensible assumption - then what keeps a mathematical culture together need not be a large-scale agreement about certain beliefs. Cultures can form when the Rousian situation appeals, even if the reasons why the situation appeals are different for different agents. If this is correct, then Rousian situations can enable mathematical cultures if they provide elements that can be appreciated by mathematicians. Folding paper, we submit, is such a Rousian situation. Paper thus enables a mathematical culture because it provides a Rousian situation.

\section{Conclusion}

In this paper, we presented folding as a material reasoning practice. As was surveyed, there was and is a sufficiently large community of mathematicians who share in the same situation. Thus, folding should be regarded as one of the mathematical reasoning practices. This reasoning practice is neither purely propositional, since it relies on certain qualities of the physical artifacts used, nor is it diagrammatic, since non-textual inference steps are not necessary in this reasoning practice. It is material because the material used justifies the constructive inference steps used in this reasoning practice. Thus, folding is a further facet of the plurality of reasoning styles used in mathematics.

For these reasons we claim that the mathematical material practice of folding enables a unique mathematical culture. This culture is distinct from diagrammatic mathematical cultures $^{52}$ because, as we showed, the modes of reasoning of folding are different from the modes of reasoning in diagrammatic reasoning cultures. Furthermore, we showed that folding does not form a part of a "mathematical modernism" culture, which in a lot of ways neglected and rejected reasoning practices of mathematics which are not purely propositional, such as drawing two-dimensional diagrams, constructing three-dimensional models or folding paper. Understood as a movement, however, mathematical modernism influences the folding culture: reasoning by folding was and is operating along the syntactical idiom propagated by mathematical modernism. The mathematicians who folded (and fold) often rely on this idiom to prove the generality of material operations.

Although many of the mathematicians who practiced paper folding as mathematical practice did not share the same beliefs regarding the aims and the epistemic strength of folding as a mathematical material practice, they shared the same situation. This situation is brought about by the physical realities of the material, which provides a structure of control for geometric constructions. Hence, considering folding as a mathematical haptic operation - as

\footnotetext{
${ }^{52}$ We can read works such as De Toffoli and Giardino's (2014) and (2016) as arguments that cultures of diagrammatic reasoning exist in contemporary mathematics.
} 
all the above-discussed mathematicians did - enabled the emergence of this material practice as a shared situation and thereby prompted, starting at least from the end of the $19^{\text {th }}$ century, the emergence of a distinctive and unique mathematical culture.

\section{Acknowledgements}

The authors are grateful to event audiences in Nancy and Oxford for their patience and helpful questions. We are also thankful to the Novembertagung in the History and Philosophy of Mathematics for providing a point of contact for the first and second author. Valeria Giardino, Yacin Hamami, Brendan Larvor, and Lisa Rougetet we thank for their insightful remarks. Research for this paper by the second author has been funded by the Research Foundation Flanders (FWO), project G056716N.

\section{Bibliography}

Ahrens, W. (1901). Mathematische Unterhaltungen und Spiele. Leipzig: B. G. Teubner.

Alperin, R. C., Lang, R. J. (2009). One-, Two-, and Multi-Fold Origami Axioms. In R. J. Lang (Ed.), Origami4: Fourth International Meeting of Origami Science, Mathematics, and Education (pp. 371-393). Natick, Massachusetts: A. K. Peters.

Avigad, J., Dean, E., Mumma, J. (2009). A Formal System for Euclid's Elements. The Review of Symbolic Logic, 2, 700-768.

Banks, J. A., McGee Banks, C. A. (1989). Multicultural education. Needham Heights, MA: Allyn \& Bacon.

Beloch, M. P. (1934). Alcune applicazioni del metodo del ripiegamento della carta di Sundara-Row. Atti dell'Acc. di Scienze, Mediche, Naturali e Matematiche di Ferrara, Serie II, $11,186-189$.

Bern, M., Demaine, E. D., Eppstein, D., Kuo, E., Mantler, A., Snoeyink, J. (2003).

Ununfoldable polyhedra with convex faces. Computational Geometry, 24(2), 51-62.

Bern, M., Hayes, B. (1996). The complexity of flat origami. Proceedings of the 7th Annual ACM-SIAM Symposium on Discrete Algorithms, 175-183.

Bos, H. (2001). Redefining Geometrical Exactness. New York: Springer.

Carter, J. (2010). Diagrams and Proofs in Analysis. International Studies in the Philosophy of Science, 24(1), 1-14.

De Toffoli, S., Giardino, V. (2014). Forms and Roles of Knot Diagrams. Erkenntnis, 79(4), $829-842$. 
De Toffoli, S., Giardino, V. (2016). Envisioning Transformations - The Practice of Topology. In B. Larvor (Ed.), Mathematical Cultures. The London Meetings 2012-2014 (pp. 25-50). Basel: Birkhäuser.

Demaine, E. D., Demaine, M. L., Lubiw, A. (1999). Folding and One Straight Cut Suffice. Proceedings of the $10^{\text {th }}$ Annual ACM-SIAM Symp. Discrete Algorithm, 891-892.

Demaine, E. D., O’Rourke, J. (2007). Geometric Folding Algorithms: Linkages, Origami, Polyhedra. Cambridge: Cambridge University Press.

Ferreirós, J. (2016). Mathematical Knowledge and the Interplay of Practices. Princeton: Princeton University Press.

Friedman, M. (2016). Two Beginnings of Geometry and Folding: Hermann Wiener and Sundara Row. Journal of the British Society for the History of Mathematics, 31(1), 52-68.

Friedman, M. (2018). On the History of Folding in Mathematics: A Mathematization of the Margins. Basel: Birkhäuser.

Frigerio, E., Huzita, H. (1989). A Possible Example of System Expansion in Origami Geometry. In H. Huzita (Ed.), Proceedings of the First International Meeting of Origami, Science and Technology (pp. 53-69). Ferrara: Comune di Ferrara and Centro Origami Diffusion.

Gallivan, B. C. (2002). How to fold paper in half twelve times (an "impossible challenge" solved and explained). Pomona California: Historical Society of Pomona Valley.

Giaquinto, M. (2007). Visual Thinking in Mathematics. Oxford: Oxford University Press.

Gray, J. (2006). Modern Mathematics as a Cultural Phenomenon. In J. Ferreirós, J. Gray (Eds.), The Architecture of Modern Mathematics (pp. 371-396). Oxford: Oxford University Press.

Hull, T. C. (2011). Solving Cubics With Creases: The Work of Beloch and Lill. The American Mathematical Monthly, 118(4), 307-315.

Huzita, H. (1989). Axiomatic Development of Origami Geometry. In H. Huzita (Ed.), Proceedings of the First International Meeting of Origami, Science and Technology (pp. 143158). Ferrara: Comune di Ferrara and Centro Origami Diffusion.

Huzita, H., Scimemi, B. (1989). The Algebra of Paper-Folding (Origami). In H. Huzita (Ed.) Proceedings of the First International Meeting of Origami, Science and Technology (pp. 215222). Ferrara: Comune di Ferrara and Centro Origami Diffusion.

Justin, J. (1989 [1986]). Résolution par le pliage de l'équation du troisième degré et applications géométriques. L'Ouvert: Journal of the APMEP of Alsace and the IREM of Strasbourg, 42, 9-19; reprinted in: H. Huzita (Ed.), Proceedings of the First International Meeting of Origami Science and Technology (pp. 251-261). Ferrara: Comune di Ferrara and Centro Origami Diffusion. 
Klein, F. (1895). Vorträge über ausgewählte Fragen der Elementargeometrie. Leipzig: Teubner.

Klein, F. (1897). Famous Problems of Elementary Geometry (trans.: Beman, W. W., Smith, D. E.). New York: Ginn and Co.

Kroeber, A. L., Kluckhohn, C. (1952). Culture: A critical review of concepts and definitions. Harvard University Peabody Museum of American Archeology and Ethnology, Papers 47.

Larvor, B. (2012). How to think about informal proofs. Synthese, 187(2), 715-730.

Larvor, B. (Ed.) (2016). Mathematical Cultures. The London Meetings 2012-2014. Basel: Birkhäuser.

Larvor, B. (2017). From Euclidean Geometry to knots and nets, Synthese, https://doi.org/10.1007/s11229-017-1558-x

Leitgeb, H. (2009). On Formal and Informal Provability. In O. Bueno, Ø. Linnebo (Eds.), New Waves in Philosophy of Mathematics (pp. 263-299). Hampshire, New York: Palgrave Macmillan.

Mancosu, P. (Ed.) (2008). The Philosophy of Mathematical Practice. Oxford: Oxford University Press.

Manders, K. (2008). The Euclidean Diagram (1995). In P. Mancosu (Ed.), The Philosophy of Mathematical Practice (pp. 80-133). Oxford: Oxford University Press.

Mehrtens, H. (1990). Moderne-Sprache-Mathematik. Eine Geschichte des Streits um die Grundlagen der Disziplin und des Subjekts formaler Systeme. Frankfurt am Main: Suhrkamp.

Mehrtens, H. (2004). "Mathematical Models.” In de Chadarevian, S., Hopwood, N. (Eds.), Models: The Third Dimension of Science, 276-306. Stanford: Stanford University Press.

Netz, R. (1999). The Shaping of Deduction in Greek Mathematics: A Study in Cognitive History. Cambridge: Cambridge University Press.

Netz, R. (2017). Mathematical Concepts? The View from Ancient History. In E. De Freitas, N. Sinclair, A. Coles, (Eds.), What is a Mathematical Concept? Cambridge: Cambridge University Press, 36-52.

Oswald, N. (2015). Adolf Hurwitz faltet Papier. Mathematische Semesterberichte, 62(2), $123-130$.

Plutarch (1961). Moralia, vol. 9, Loeb Classical Library (trans.: Minar, E. L. Jr., Sandbach, F. H., Helmbold, W. C.). London: Heinemann.

Richerson, P. J., Boyd, R. (2005). Not by Genes Alone: How Culture Transformed Human Evolution. Chicago: The University of Chicago Press. 
Rouse, J. (1996). Engaging Science: How to Understand Its Practices Philosophically. Ithaca, London: Cornell University Press.

Row, S. T. (1893). Geometrical Exercises in Paper Folding. Madras: Addison.

Row, S. T. (1901). Geometrical Exercises in Paper Folding (Eds.: Beman, W. W./Smith, D. E.). Chicago: The Open Court.

Rupp, C. A. (1924). On a Transformation by Paper Folding. Amer. Math. Monthly, 31, 432435 .

Stone, M., Stojnic, U. (2015). Meaning and Demonstration. Review of Philosophy and Psychology, 6(1), 69-97.

Tylor, E. (1871). Primitive Culture: researches into the development of mythology, philosophy, religion, art, and custom. Vol. 2. London: J. Murray.

Young, G. C., Young, W. H. (1905). The First Book of Geometry. New York: Chelsea Publishing Company. 